\title{
A 3-D Global Earth-Ionosphere FDTD Model Including an Anisotropic Magnetized Plasma Ionosphere
}

\author{
Yaxin Yu, Member, IEEE, Jiajun Niu, Student Member, IEEE, and Jamesina J. Simpson, Member, IEEE
}

\begin{abstract}
A 3-D global Earth-ionosphere finite-difference time-domain (FDTD) model is introduced that includes for the first time an anisotropic magnetized cold plasma ionosphere. All previous global FDTD Earth-ionosphere models to date have employed an isotropic conductivity profile. To generate the new model, a previously validated and published 3-D Cartesian magnetized cold plasma algorithm is adapted to the global latitude-longitude FDTD mesh that involves trapezoidal cells, merging of cells in the Polar regions, and triangular cells at the Poles. The global geomagnetic field, ionospheric particle densities and collision frequencies, as well as the Earth's topographic and bathymetric data are all mapped onto the global space grid. After a local high-resolution validation that demonstrates correct calculations of electromagnetic propagation in magnetized plasma, another numerical study is performed to validate the model on a global scale. This new model opens doors to a wide variety of advanced modeling for higher frequency and higher altitude electromagnetic phenomena and represents a paradigm shift from the commonly used ray-tracing codes. It also provides the opportunity to couple FDTD Earth-ionosphere models to other geophysical models, such as the Naval Research Lab's SAMI3, to yield a multiphysics simulator.
\end{abstract}

Index Terms -Earth, electromagnetic wave propagation, finitedifference time-domain (FDTD) method, ionosphere, magnetized cold plasma.

\section{INTRODUCTION}

$\mathbf{F}$ OR almost two decades, the finite-difference time-domain (FDTD) [1], [2] method has been applied towards modeling electromagnetic (EM) wave propagation within the Earthionosphere system [3], [4]. Initially, due to constraints of available computational resources, 2-D FDTD models were generated and employed to study, for example, long-range propagation [5], [6], propagation from lightning [7], D-region perturbation induced by solar flare [8], and energy spectrum of ce-

Manuscript received July 01, 2011; revised January 07, 2012; accepted February 15,2012 . Date of publication May 01,2012 ; date of current version July 02, 2012. Computing support for this work was provided by the New Mexico Computing Applications Center (NMCAC) and the University of New Mexico High Performance Computing Center (HPCC). This work was supported by a National Science Foundation CAREER Award, Grant \#0955404.

$\mathrm{Y}$. Yu is with the Institute of High Performance Computing, A*STAR, 138632, Singapore.

J. Niu is with the Electrical and Computer Engineering Department at the University of New Mexico, Albuquerque, NM 87131 USA.

J. J. Simpson is with the Electrical and Computer Engineering Department at the University of Utah, Salt Lake City, UT 84112 USA (e-mail: simpson@ece. utah.edu).

Digital Object Identifier 10.1109/TAP.2012.2196937 lestial gamma-ray bursts [9], etc. As computing resources continued to improve, global 3-D FDTD models began to appear for extremely low-frequency (ELF) propagation within the Earthionosphere system. These global models were applied, for example, towards calculations of propagation from lightning [10], [11], Schumann resonances [12], remote sensing of oil fields [13], remote sensing of ionospheric disturbances [14], modeling hypothesized EM earthquake precursors [15], and space weather effects on the operation of power grids [16]. References [3] and [4] provide a thorough summary of all FDTD local and global models of the Earth-ionosphere system up until the time of their respective publication dates.

Note that all of the global models to date have utilized an isotropic conductivity profile to represent the ionosphere. As a result, the ionosphere is treated as a simple isotropic medium that ignores the influence of the geomagnetic field. This appears to be adequate in calculating the average propagation of EM waves below $\sim 100 \mathrm{~km}$ altitude and at frequencies less than $\sim 1 \mathrm{kHz}$ over thousands of kilometers [4]. However, by not including effects introduced by the anisotropic geomagnetic field, these models are not capable of modeling Faraday rotation in the ionosphere [17], whistler wave injection [18], and lightning-induced electron precipitation [19], for example.

This paper concerns the advancement of the global FDTD Earth-ionosphere models to account for the physics introduced by the magnetized ionospheric plasma. Incorporating this modeling capability will permit higher frequency (above $\sim 1 \mathrm{kHz}$ ) and higher altitude (above an altitude of $\sim 100 \mathrm{~km}$ ) propagation studies than permitted by all of the previous global models involving isotropic conductivity profiles. As such, by being able to account for the geomagnetic field, ionospheric particle densities and collision frequencies, the Earth's topography, oceans, and lithosphere layering, as well as any antennas and obstacles (manmade or natural), these models represent a paradigm shift from ray-tracing codes. Furthermore, this advancement introduces the possibility of coupling the FDTD Maxwell's equations models with models of other geophysical processes to yield multiphysics simulators. For example, FDTD models having a magnetized ionospheric plasma can be coupled with the Naval Research Lab's SAMI3 (SAMI3 is also a model of the ionosphere), which solves the 5-moment set of transport equations of magnetized plasma and also accounts for photoionization and chemical processes [20].

As such, we report here what we believe to be the first global FDTD Earth-ionosphere model that includes a magnetized ionospheric plasma algorithm. To generate this model, the previ- 


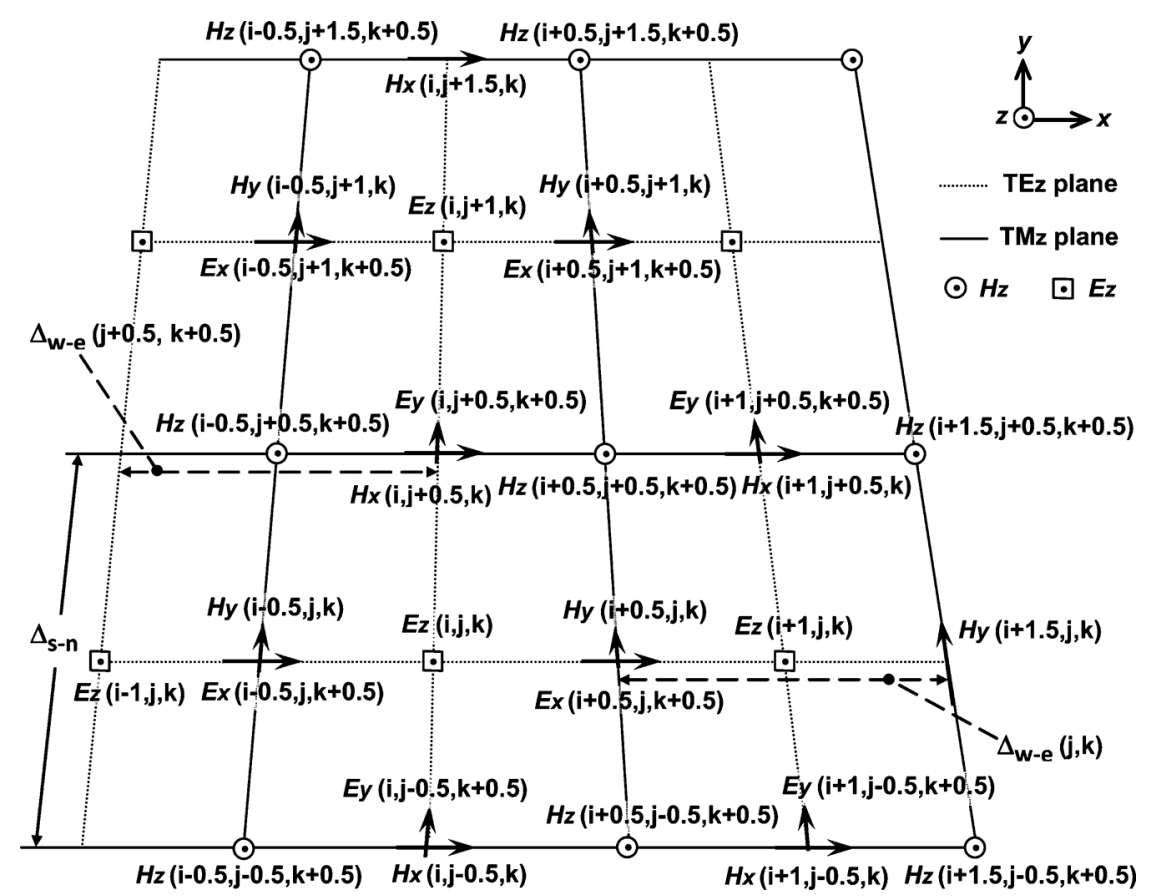

Fig. 1. Nonmerging latitude-longitude grid cells for the Northern hemisphere (a $\mathrm{TE}_{z}$ plane is superimposed onto a $\mathrm{TM}_{z}$ plane so that both the $\mathrm{k}$ and $k+0.5$ components are illustrated).

ously validated 3-D Cartesian-coordinate cold plasma model of [21] is taken and adapted to the latitude-longitude global model of [22]. Here, the latitude-longitude model is chosen over the global geodesic model of [23] for ease of implementation.

The 3-D Cartesian cold plasma model of [21] is based on the 2-D cylindrical-coordinate plasma algorithm of [24]. This algorithm has the capability to simulate wave behaviors in cold plasma under applied magnetic fields of arbitrary direction and magnitude. Plasma effects contributed by electrons, positive, and negative ions may all be included. Note that a magnetic-field-independent absorbing boundary condition $(\mathrm{ABC})$ has been recently developed to truncate computational domains that employ the plasma algorithm [25]. This ABC may be incorporated on a global scale on the upper radial (or vertical) side of the computational domain if needed, or it may be useful for terminating localized (in the latitude-longitude direction) models of the Earth-ionosphere system.

One of the most important characteristics of the plasma algorithms of [21] and [24] is that, for modeling the ionosphere, they do not introduce a late-time instability or require an order-of-magnitude smaller time step than that permitted by the Courant limit [2]. These were limitations found for other FDTD plasma algorithms when modeling the ionosphere, such as those in [26]-[28]. This characteristic allows for complete transformation from the 3-D Cartesian-coordinate formulation of [21] into the 3-D latitude-longitude grid structure of [22] without loss of its medium-independent stability property for highly anisotropic ionosphere media.

The remainder of this paper is structured as follows. In Section II, an algorithm overview is provided for the new 3-D global FDTD model based on [22] but upgraded to include an adapted version of the magnetized cold plasma algorithm of [21]. Section III then details the construction process of the global latitude-longitude gyrotropic cold plasma algorithm, and Section IV shows its accuracy by comparing the local simulation results to those in [21]. Section V describes the validation study for impulsive ELF propagation using the newly-developed global plasma-ionosphere model, and Section VI provides the validation test results. Finally, Section VII concludes with a discussion of ongoing work and potential applications of this model.

\section{ALGORITHM OVERVIEW}

The updating scheme for each magnetic field intensity $H$ component of the global model is identical to those presented in [22], regardless of its position within the ionosphere, atmosphere, oceans, or lithosphere. The updating scheme for each electric field $E$ component in the atmosphere, ocean and lithosphere portions are also taken here to be identical to those shown in [22]. The reader is referred to [22] for a more detailed description for these "regular" updates.

The $E$-fields within the magnetized cold plasma region require an altered updating scheme. Specifically, the original iteration set (33) in [21] for implementing the magnetized plasma in Cartesian-coordinate must be adapted to accommodate the global latitude-longitude mesh of [22]. Equation (1) presents the resulting time-domain expression for calculating the field components of $E$ and electron current density $J_{e}$ for altitudes within the cold plasma ionosphere. Here, only the effects of electrons within the ionospheric plasma are considered; however, (1) can 
be easily expanded to include the effects of positive and negative ions by following the methodology of [21]

$$
\left[\begin{array}{c}
E_{x}^{n+1} \\
E_{y}^{n+1} \\
E_{z}^{n+1} \\
J_{e x}^{n+1} \\
J_{e y}^{n+1} \\
J_{e z}^{n+1}
\end{array}\right]=D \cdot\left[\begin{array}{c}
E_{x}^{n} \\
E_{y}^{n} \\
E_{z}^{n} \\
J_{e x}^{n} \\
J_{e y}^{n} \\
J_{e z}^{n}
\end{array}\right]+U \cdot\left[\begin{array}{c}
\left(\frac{\Delta H_{z}}{\Delta_{s-n}}\right)^{n+1 / 2} \\
\left(\frac{\Delta H_{y}}{\Delta_{r}}\right)^{n+1 / 2} \\
\left(\frac{\Delta H_{x}}{\Delta_{r}}\right)^{n+1 / 2} \\
\left(\frac{\Delta H_{z}}{\Delta_{w-e}}\right)^{n+1 / 2} \\
\left(\frac{\Delta H_{y}}{\Delta_{w-e}}\right)^{n+1 / 2} \\
\left(\frac{\Delta\left(H_{x} \Delta_{w-e}\right)}{S}\right)^{n+1 / 2}
\end{array}\right]
$$

As defined in [22], $\Delta_{w-e}$ and $\Delta_{s-n}$ are the West-East and South-North span of each trapezoidal or triangular grid cell, respectively. $\Delta_{r}$ is the cell span in the radial direction. $S$ is the corresponding trapezoidal or triangular cell area centered at the grid component being updated.

$\mathrm{D}$ (expressed as $A^{-1} B$ in [21]) and $\mathrm{U}$ (expressed as $A^{-1} C$ in [21]) are the corresponding $6 \times 6$ iteration coefficient matrices needed for implementing the plasma system. The matrix entries in $\mathrm{D}$ and $\mathrm{U}$ depend on the modeling parameters as well as the ionospheric plasma properties (particle densities, collision frequencies, and geomagnetic field components) that vary with altitude ( $k$-direction) and position $(i, j)$ around the Earth.

This magnetized plasma algorithm adopts the so-called $E-J$ collocation technique that collocates the current density components $J_{e x}, J_{e y}, J_{e z}$ in both time and space with their corresponding $E_{x}, E_{y}$, and $E_{z}$, respectively. As such, the updating schemes of the $J$ components are analogous to their corresponding $E$-field counterparts and, therefore, will not be separately discussed in this paper.

According to (1), the time-domain updating equations for $E_{x}$, $E_{y}$, and $E_{z}$ in a magnetized cold plasma can be expressed as

$$
\begin{aligned}
E_{x}^{n+1}= & D^{<1,1>} E_{x}^{n}+D^{<1,2>} \overline{E_{y}^{n}}+D^{<1,3>} \overline{E_{z}^{n}} \\
& +D^{<1,4>} J_{e x}^{n}+D^{<1,5>} \overline{J_{e y}^{n}} \\
& +D^{<1,6>} \overline{J_{e z}^{n}}+U^{<1,1>}\left(\frac{\Delta H_{z}}{\Delta_{s-n}}\right)^{n+1 / 2} \\
& +U^{<1,2>} \frac{\left(\frac{\Delta H_{y}}{\Delta_{r}}\right)^{n+1 / 2}}{\left(\frac{\Delta H_{x}}{\Delta_{r}}\right)^{n+1 / 2}} \\
& +U^{<1,3>} \\
& +U^{<1,4>} \frac{\left(\frac{\Delta H_{z}}{\Delta_{w-e}}\right)^{n+1 / 2}}{\left(\frac{\Delta H_{y}}{\Delta_{w-e}}\right)^{n+1 / 2}} \\
& +U^{<1,5>} \\
& +U^{<1,6>} \frac{\left(\frac{\Delta\left(H_{x} \Delta_{w-e}\right)}{S}\right)^{n+1 / 2}}{S}
\end{aligned}
$$

$$
\begin{aligned}
& E_{y}^{n+1}=D^{<2,1>} \overline{E_{x}^{n}}+D^{<2,2>} E_{y}^{n}+D^{<2,3>} \overline{E_{z}^{n}} \\
& +D^{<2,4>} \overline{J_{e x}^{n}}+D^{<2,5>} J_{e y}^{n}+D^{<2,6>} \overline{J_{e z}^{n}} \\
& +U^{<2,1>} \overline{\left(\frac{\Delta H_{z}}{\Delta_{s-n}}\right)^{n+1 / 2}} \\
& +U^{<2,2>} \overline{\left(\frac{\Delta H_{y}}{\Delta_{r}}\right)^{n+1 / 2}} \\
& +U^{<2,3>}\left(\frac{\Delta H_{x}}{\Delta_{r}}\right)^{n+1 / 2} \\
& +U^{<2,4>}\left(\frac{\Delta H_{z}}{\Delta_{w-e}}\right)^{n+1 / 2} \\
& +U^{<2,5>} \overline{\left(\frac{\Delta H_{y}}{\Delta_{w-e}}\right)^{n+1 / 2}} \\
& +U^{<2,6>} \overline{\left(\frac{\Delta\left(H_{x} \Delta_{w-e}\right)}{S}\right)^{n+1 / 2}} \\
& E_{z}^{n+1}=D^{<3,1>} \overline{E_{x}^{n}}+D^{<3,2>} \overline{E_{y}^{n}}+D^{<3,3>} E_{z}^{n} \\
& +D^{<3,4>} \overline{J_{e x}^{n}}+D^{<3,5>} \overline{J_{e y}^{n}}+D^{<3,6>} J_{e z}^{n} \\
& +U^{<3,1>} \overline{\left(\frac{\Delta H_{z}}{\Delta_{s-n}}\right)^{n+1 / 2}} \\
& +U^{<3,2>} \overline{\left(\frac{\Delta H_{y}}{\Delta_{r}}\right)^{n+1 / 2}} \\
& +U^{<3,3>} \overline{\left(\frac{\Delta H_{x}}{\Delta_{r}}\right)^{n+1 / 2}} \\
& +U^{<3,4>} \overline{\left(\frac{\Delta H_{z}}{\Delta_{w-e}}\right)^{n+1 / 2}} \\
& +U^{<3,5>}\left(\frac{\Delta H_{y}}{\Delta_{w-e}}\right)^{n+1 / 2} \\
& +U^{<3,6>}\left(\frac{\Delta\left(H_{x} \Delta_{w-e}\right)}{S}\right)^{n+1 / 2}
\end{aligned}
$$

where the superscript $\langle x, y\rangle$ denotes the corresponding index of the individual matrix element of $\mathrm{D}$ and $\mathrm{U}$.

As discussed in [21], to update $E_{x}, E_{y}$, and $E_{z}$ within the magnetized cold plasma region of the global FDTD algorithm using (2)-(4), all of the six $E$ and $J_{e}$ field quantities and the six $H$-derivatives at previous time-step must be evaluated at the same grid position of the corresponding to-be-updated $E$-field component. This requires implementation of spatial-averaging as in [21] to be used for all of the spatially noncollocated state variables and derivatives in the latitude-longitude mesh. A bar over the field components within (2)-(4) denotes those components must be spatially averaged to perform the indicated calculation.

\section{Global Magnetized Plasma Algorithm Details}

\section{A. Nonmerging Trapezoidal Grid Cells}

Fig. 1 illustrates nonmerging latitude-longitude grid cells. To show the relative lateral positions of the different grid components, a $\mathrm{TE}_{z}$ plane is superimposed onto a $\mathrm{TM}_{z}$ plane. In the 
actual FDTD model, these two planes are separated by a distance of $\Delta_{r} / 2$ in the radial $k$-direction.

To be consistent with reference [22], $E_{x}, E_{y}$, and $E_{z}$ are calculated at $(i+0.5, j, k+0.5),(i, j+0.5, k+0.5)$ and $(\mathrm{i}, \mathrm{j}$, k), respectively. Referring to Fig. $1, E_{x}(i+0.5, j, k+0.5)$ can be updated through (2) with the noncollocated state variables and derivatives calculated by the following spatial-averaging expressions [see (5)-(10), shown at the bottom of the page].

Substituting (5)-(10) into (2), we can obtain the $E_{x}$ updating equation for regular trapezoidal grid cells. Note that the spatialaveraging expressions of the $J$ components are similar to their $E$-field counterparts.

Following an analogous process, $E_{y}$ and $E_{z}$ updates can be formulated for the nonmerging trapezoidal cells. Due to space constraints, these update equations will not be included here.

\section{B. Merging Trapezoidal Grid Cells}

As pointed out in [22], the geometrical eccentricity of each individual cell becomes quite large near the North and South Poles, thereby degrading the numerical stability limit and efficiency of the global FDTD algorithm. To mitigate this problem, an adaptive cell-combining technique involving merging pairs of adjacent cells in the West-East direction is applied in this global model when approaching the polar-regions. This process can be repeated several times based on the user-specified maximum allowable eccentricity.

Fig. 2 illustrates the FDTD mesh containing merged trapezoidal grid cells when approaching the North-Pole region. As for Fig. 1, a $\mathrm{TE}_{z}$ plane is superimposed onto a $\mathrm{TM}_{z}$ plane although these planes are actually separated in the radial $k$-direction by $\Delta_{r} / 2$. Updates for the $E$-field components within merging cells follow the same procedure as for nonmerging cells, except that special care must be taken when performing spatial averaging.

For example, referring to Fig. $2, E_{z}(i, j, k)$ at the center of the merged $\mathrm{TM}_{z}$-plane trapezoidal cell can be updated through (4) using (11)-(16), shown at the bottom of the next page.

In a similar manner, the $E_{x}$ components can be updated via analogous spatial averaging as for the $E_{z}$ components. On the other hand, due to the cell-combining operations, the spatial-averaging schemes for the $E_{y}$ components located at varying grid positions are different (depending on whether the component is located above or below the merging interface, and depending on if it has an even or odd spatial $i$-index).

$$
\begin{aligned}
& \text { overline }\left.E_{y}\right|_{i+0.5, j, k+0.5} ^{n}=\frac{\left(\begin{array}{c}
\left.E_{y}\right|_{i, j+0.5, k+0.5} ^{n}+\left.E_{y}\right|_{i+1, j+0.5, k+0.5} ^{n}+ \\
\left.E_{y}\right|_{i+1, j-0.5, k+0.5} ^{n}+\left.E_{y}\right|_{i, j-0.5, k+0.5} ^{n}
\end{array}\right)}{4} \\
& \overline{\left.E_{z}\right|_{i+0.5, j, k+0.5} ^{n}}=\frac{\left(\left.E_{z}\right|_{i, j, k} ^{n}+\left.E_{z}\right|_{i+1, j, k} ^{n}+\left.E_{z}\right|_{i, j, k+1} ^{n}+\left.E_{z}\right|_{i+1, j, k+1} ^{n}\right)}{4}
\end{aligned}
$$

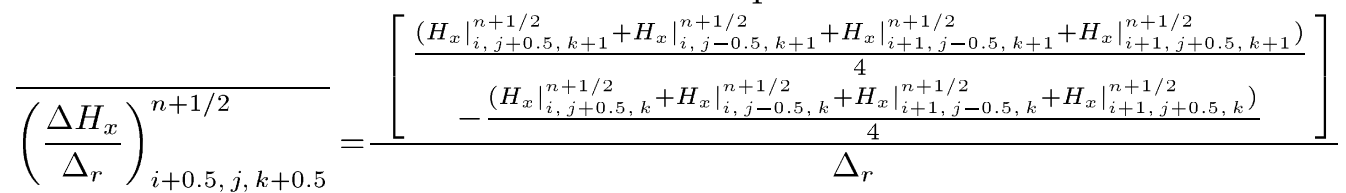

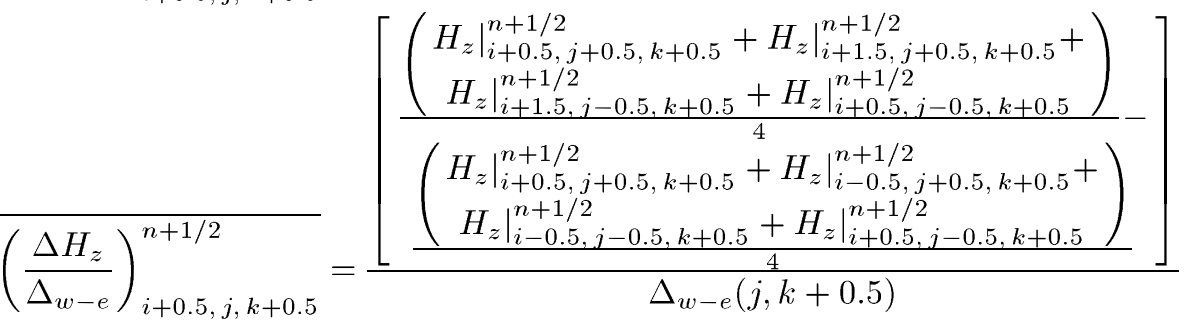

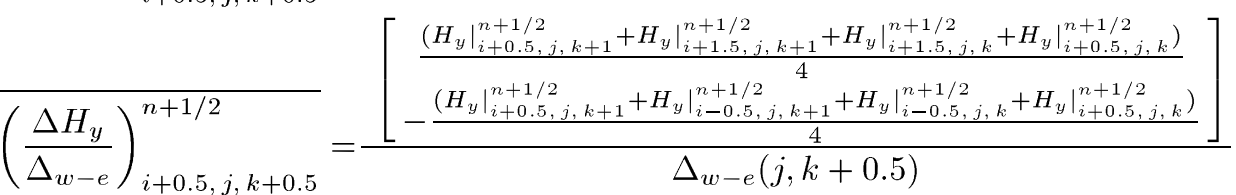

$$
\begin{aligned}
& =\frac{\left[\begin{array}{c}
\Delta_{w-e}(j+0.5, k+0.5) \cdot \frac{\left(\left.H_{x}\right|_{i+1, j+0.5, k+1} ^{n+1 / 2}+\left.H_{x}\right|_{i+1, j+0.5, k} ^{n+1 / 2}+\left.H_{x}\right|_{i, j+0.5, k+1} ^{n+1 / 2}+\left.H_{x}\right|_{i, j+0.5, k} ^{n+1 / 2}\right)}{4} \\
-\Delta_{w-e}(j-0.5, k+0.5) \cdot \frac{\left(\left.H_{x}\right|_{i+1, j-0.5, k+1} ^{n+1 / 2}+\left.H_{x}\right|_{i+1, j-0.5, k} ^{n+1 / 2}+\left.H_{x}\right|_{i, j-0.5, k+1} ^{n+1 / 2}+\left.H_{x}\right|_{i, j-0.5, k} ^{n+1 / 2}\right)}{4}
\end{array}\right]}{S(j, k+0.5)}
\end{aligned}
$$




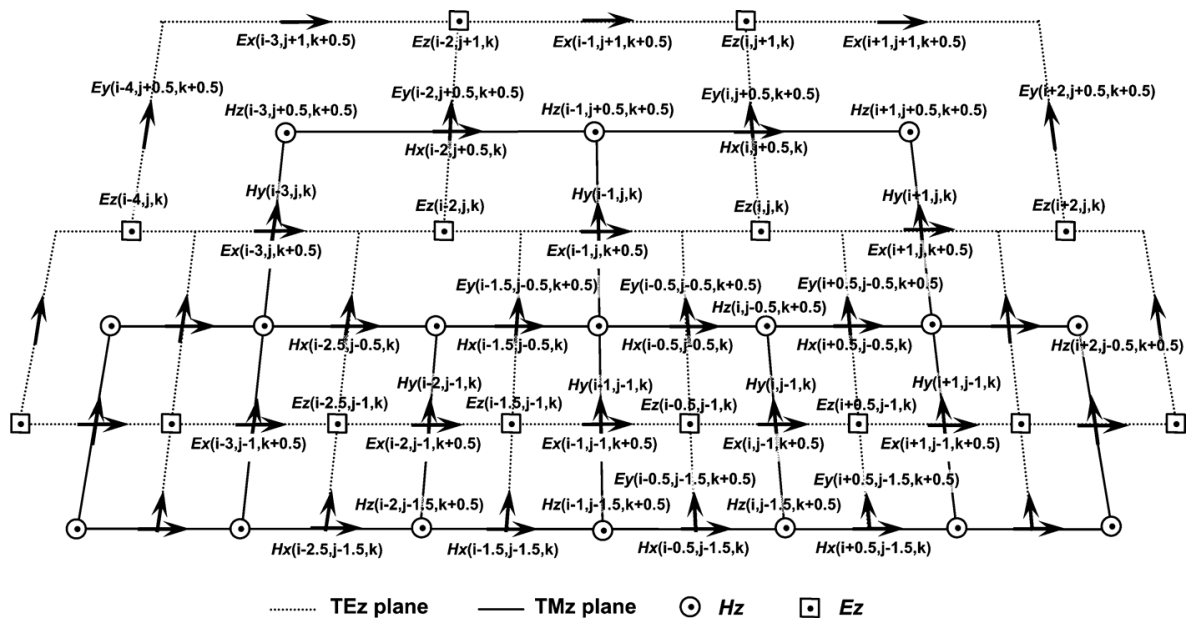

Fig. 2. Merging trapezoidal grid cells for the Northern hemisphere of the global model (a $\mathrm{TE}_{z}$ plane is superimposed onto a $\mathrm{TM}_{z}$ plane so that both the $\mathrm{k}$ and $k+0.5$ components are illustrated).

For example, referring to Fig. $2, E_{y}(i, j+0.5, k+0.5)$ involves a regular updating scheme corresponding to nonmerging trapezoidal cells, except for the following components [see (17)-(20), shown at the bottom of the next page], whereas the

$$
\begin{aligned}
& \overline{\left.E_{x}\right|_{i, j, k} ^{n}}=\frac{\left(\begin{array}{c}
\left.E_{x}\right|_{i-1, j, k+0.5} ^{n}+\left.E_{x}\right|_{i+1, j, k+0.5} ^{n}+ \\
\left.E_{x}\right|_{i-1, j, k-0.5} ^{n}+\left.E_{x}\right|_{i+1, j, k-0.5} ^{n}
\end{array}\right)}{4} \\
& \overline{\left.E_{y}\right|_{i, j, k} ^{n}}=\frac{\left(\begin{array}{c}
\frac{\left(\left.E_{y}\right|_{i-0.5, j-0.5, k+0.5} ^{n}+\left.E_{y}\right|_{i+0.5, j-0.5, k+0.5} ^{n}\right)}{2} \\
+\left.E_{y}\right|_{i, j+0.5, k+0.5} ^{n}+\left.E_{y}\right|_{i, j+0.5, k-0.5} ^{n}+ \\
\frac{\left(\left.E_{y}\right|_{i-0.5, j-0.5, k-0.5} ^{n}+\left.E_{y}\right|_{i+0.5, j-0.5, k-0.5} ^{n}\right)}{2}
\end{array}\right)}{4} \\
& \overline{\left(\frac{\Delta H_{z}}{\Delta_{s-n}}\right)_{i, j, k}^{n+1 / 2}}=\frac{\left[\begin{array}{c}
\left(\begin{array}{c}
\left.H_{z}\right|_{i-1, j+0.5, k+0.5} ^{n+1 / 2}+\left.H_{z}\right|_{i+1, j+0.5, k+0.5} ^{n+1 / 2} \\
\left.H_{z}\right|_{i-1, j+0.5, k-0.5} ^{n+1 / 2}+\left.H_{z}\right|_{i+1, j+0.5, k-0.5} ^{n+1 / 2}
\end{array}\right) \\
4 \\
-\frac{\left(\left.H_{z}\right|_{i, j-0.5, k+0.5} ^{n+1 / 2}+\left.H_{z}\right|_{i, j-0.5, k-0.5} ^{n+1 / 2}\right)}{2}
\end{array}\right]}{\Delta_{s-n}} \\
& \overline{\left(\frac{\Delta H_{y}}{\Delta_{r}}\right)_{i, j, k}^{n+1 / 2}}=\frac{\left[\begin{array}{c}
\frac{\left(\left.H_{y}\right|_{i-1, j, k} ^{n+1 / 2}+\left.H_{y}\right|_{i+1, j, k} ^{n+1 / 2}+\left.H_{y}\right|_{i-1, j, k+1} ^{n+1 / 2}+\left.H_{y}\right|_{i+1, j, k+1} ^{n+1 / 2}\right)}{4} \\
-\frac{\left(\left.H_{y} \begin{array}{l}
n+1 / 2 \\
i-1, j, k
\end{array} H_{y}\right|_{i+1, j, k} ^{n+1 / 2}+\left.H_{y}\right|_{i-1, j, k-1} ^{n+1 / 2}+\left.H_{y}\right|_{i+1, j, k-1} ^{n+1 / 2}\right)}{4}
\end{array}\right]}{\Delta_{r}}
\end{aligned}
$$

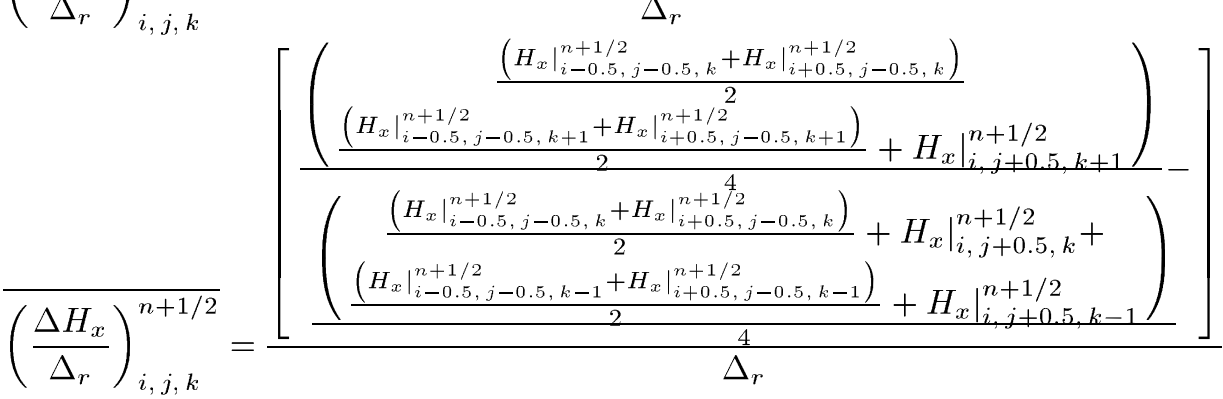

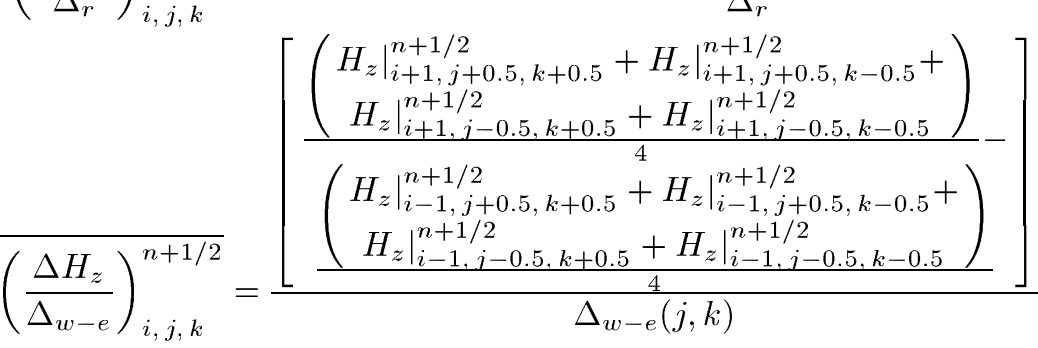




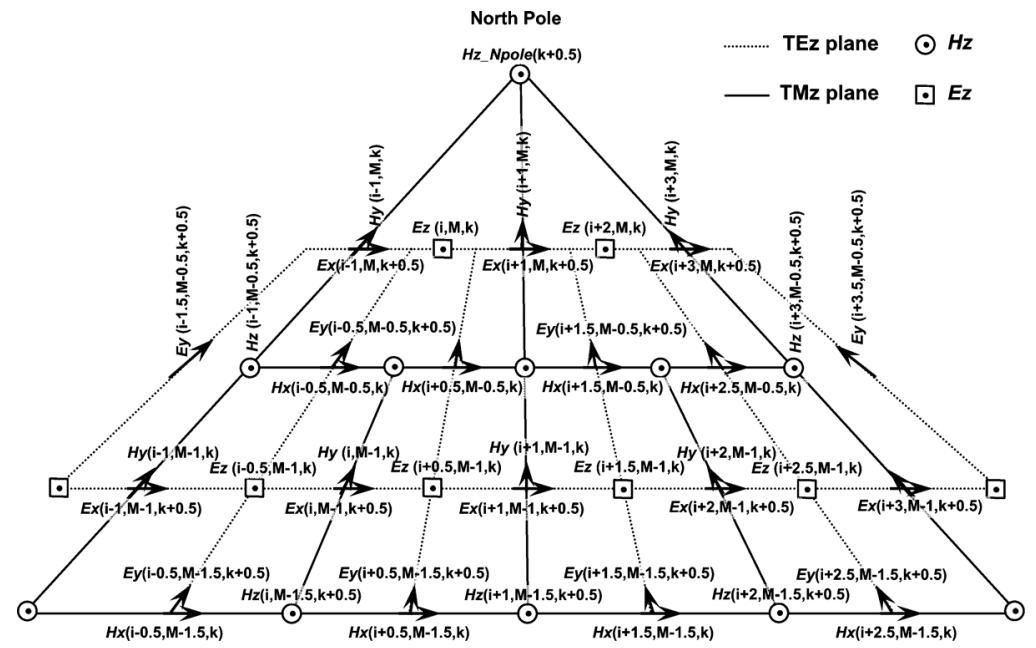

Fig. 3. Merging trapezoidal grid cells at the North Pole (a $\mathrm{TE}_{z}$ plane is superimposed onto a $\mathrm{TM}_{z}$ plane so that both the $\mathrm{k}$ and $k+0.5$ components are illustrated).

updating scheme for $E_{y}(i-0.5, j-0.5, k+0.5)$ involves a regular nonmerging trapezoidal updating scheme except for the following components [see (21)-(26), shown at the bottom of the next page].

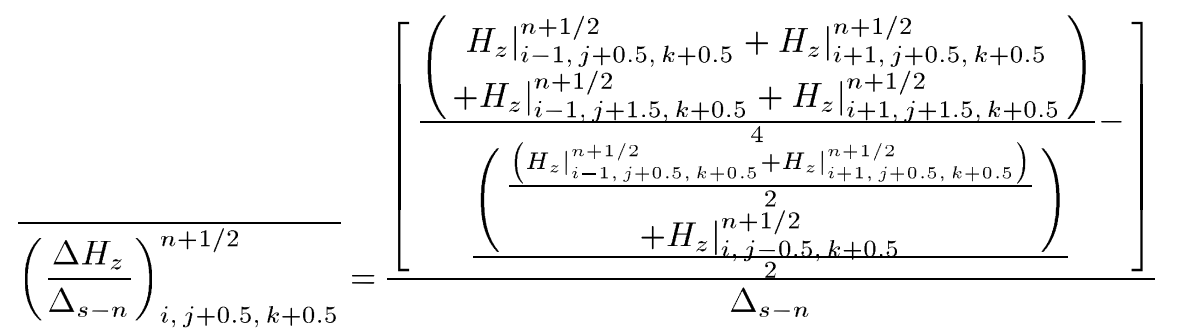

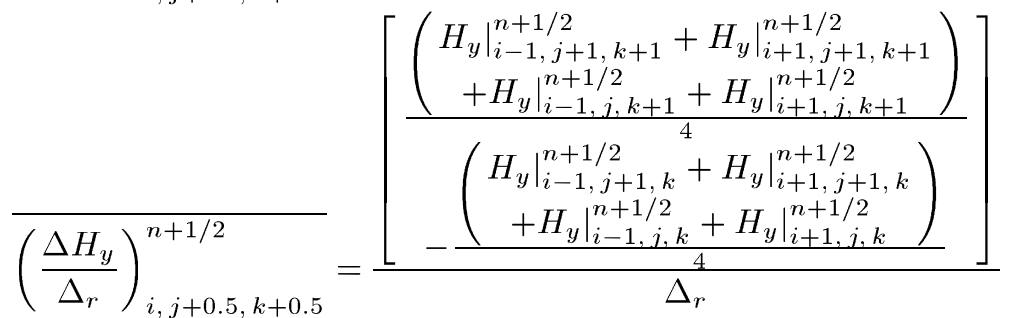

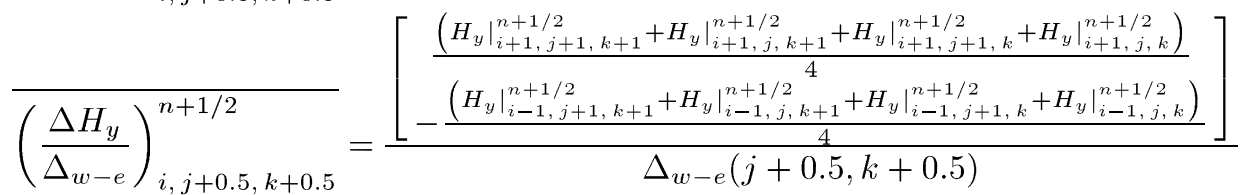

$$
\begin{aligned}
& \Delta_{w-e}(j+1, k+0.5) \text {. } \\
& \left(\begin{array}{c}
\left.H_{x}\right|_{i, j / 1.5, k+1} ^{n+1 / 2}+\left.H_{x}\right|_{i, j+1.5, k} ^{n+1 / 2}+ \\
\left.H_{x}\right|_{i, j+0.5, k+1} ^{n+1 / 2}+\left.H_{x}\right|_{i, j+0.5, k} ^{n+1 / 2}
\end{array}\right) \\
& \Delta_{w-e}(\stackrel{4}{j, k}+0.5) \text {. } \\
& \left(\begin{array}{c}
\frac{\left(\left.H_{x}\right|_{i-0.5, j-0.5, k} ^{n+1 / 2}+\left.H_{x}\right|_{i+0.5, j-0.5, k} ^{n+1 / 2}\right)}{2}+ \\
\left(\left.H_{x}\right|_{i-0.5, j-0.5, k+1} ^{n+1 / 2}+\left.H_{x}\right|_{i+0.5, j-0.5, k+1} ^{n+1 / 2}\right)
\end{array}\right) \\
& \overline{\left(\frac{\Delta\left(H_{x} \Delta_{w-e}\right)}{S}\right)_{i, j+0.5, k+0.5}^{n+1 / 2}}=\frac{\left[\frac{\left(+\left.H_{x}\right|_{i, j+0.5, k+1} ^{n+1 / 2}+\left.H_{x}\right|_{i, j+0.5, k} ^{n+1 / 2}\right.}{4}\right]}{\left[\Delta_{w-e}(j+1, k+0.5)+2 \Delta_{w-e}(j, k+0.5)\right] \cdot \frac{\Delta_{s-n}}{2}}
\end{aligned}
$$




\section{Polar Region Merging Cells}

Due to the large geometrical eccentricity of the grid cells at the North and South Poles, the row of FDTD cells directly circulating the Poles consists of a set of merging cells as shown in Fig. 3. Note that some field components are missing for updating the magnetized plasma $E$ and $J$ components directly circulating the Poles (e.g., for $E_{z}(i, M, k)$ of Fig. 3, $E_{x}$ and $E_{y}$ values to the North do not exist, but are needed in the spatial averaging calculations). So instead of using magnetized ionospheric plasma updates for these components directly circulating the Poles, nonmagnetized plasma updates are employed. That is, for the small number of components at each Pole having missing fields in their updating equations, a background magnetic field value of zero is employed, thereby eliminating any need for spatial averaging (for this case, the algorithm reduces to "normal,"

$$
\begin{aligned}
& \frac{\left.E_{x}\right|_{i-0.5, j-0.5, k+0.5} ^{n}}{n}=\frac{\left(\begin{array}{c}
\left.E_{x}\right|_{i-1, j-1, k+0.5} ^{n}+\left.E_{x}\right|_{i, j-1, k+0.5} ^{n}+\left.E_{x}\right|_{i-1, j, k+0.5} ^{n} \\
+\frac{\left(\left.E_{x}\right|_{i-1, j, k+0.5} ^{n}+\left.E_{x}\right|_{i+1, j, k+0.5} ^{n}\right)}{2}
\end{array}\right)}{4} \\
& \overline{\left.E_{z}\right|_{i-0.5, j-0.5, k+0.5} ^{n}}=\frac{\left(\begin{array}{l}
\frac{\left(\left.E_{z}\right|_{i-2, j, k} ^{n}+\left.3 E_{z}\right|_{i, j, k} ^{n}\right)}{4}+\left.E_{z}\right|_{i-0.5, j-1, k+1} ^{n}+ \\
\frac{\left(\left.E_{z}\right|_{i-2, j, k+1} ^{n}+3 E_{z}^{n}{ }_{i, j, k+1}^{n}\right)}{4}+\left.E_{z}\right|_{i-0.5, j-1, k} ^{n}
\end{array}\right)}{4}
\end{aligned}
$$

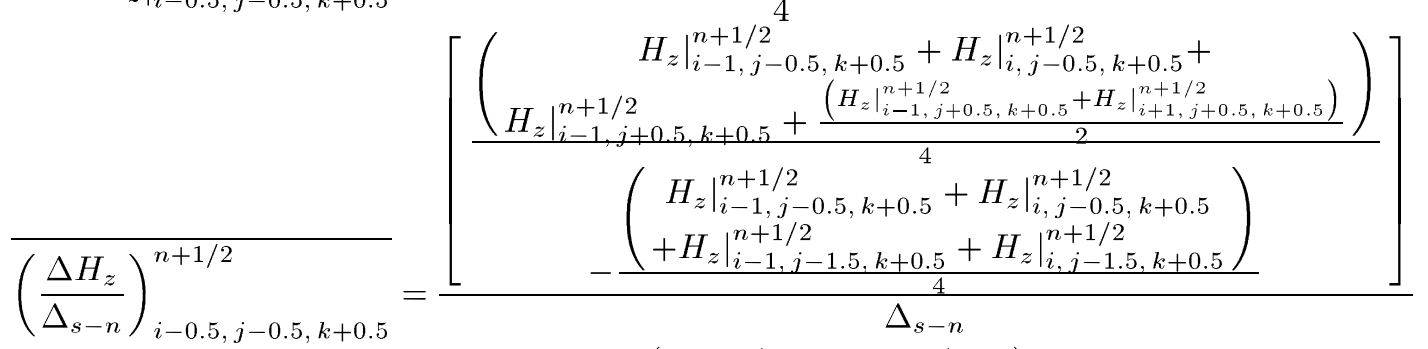

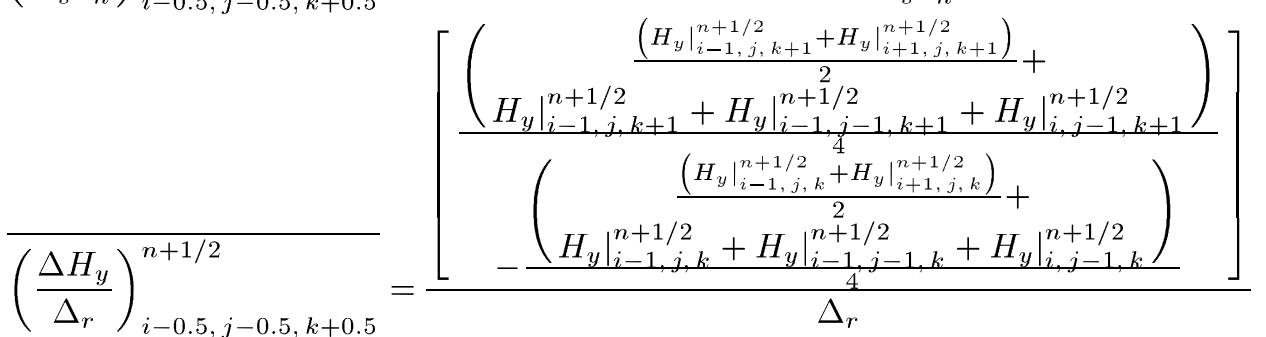

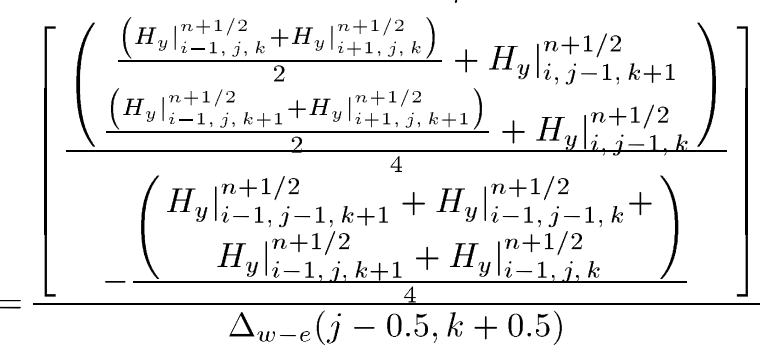

$$
\begin{aligned}
& \overline{\left(\frac{\Delta H_{y}}{\Delta_{w-e}}\right)_{i-0.5, j-0.5, k+0.5}^{n+1 / 2}}
\end{aligned}
$$

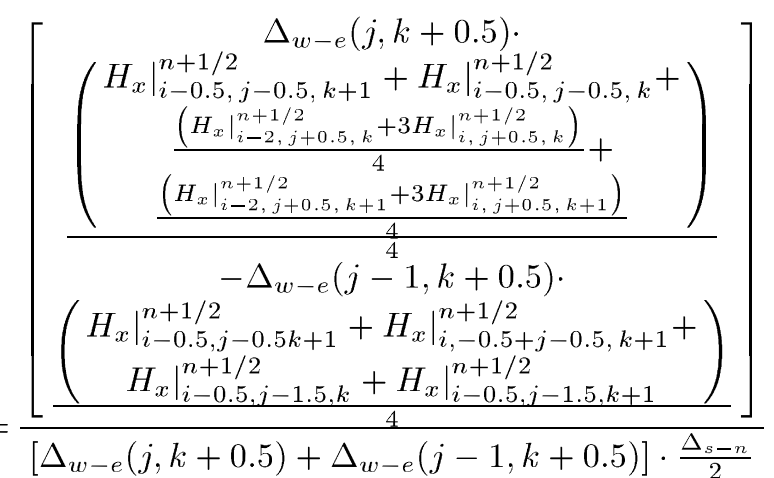


unmagnetized updates, but with the updating coefficients still depending on the particle densities and collision frequencies).

\section{Local High-Resolution, ANisotropic VALIDATION}

We first study the performance of the new 3-D trapezoidal FDTD plasma model by comparing its calculated propagation characteristics with that of the 3-D Cartesian-coordinate plasma model of [21].

As for the numerical study of Section IV of [21], the plasma is modeled as having an electron density of $1.0 \times 10^{18} \mathrm{~m}^{-3}$ (no ions) under an applied $0.06 \mathrm{~T}$ magnetic field. What is different here from the study in [21] is that instead of a Cartesian grid, the plasma medium is assumed to curve around a sphere of radius $2.6738 \mathrm{~m}$ (this small radius is chosen since the grid cells are small here as in [21] and we want here to properly test curved trapezoidal cells that are not nearly cubic cells on a large sphere). Results are obtained for propagation from the Equator to $30^{\circ} \mathrm{N}$. The radial extent of the domain is 2.6738 to $3.2138 \mathrm{~m}$. Thus, the propagation characteristics are tested here over nonuniform grid cells whose sizes vary from $1.38 \times 1.20 \times 1 \mathrm{~mm}$ to $1 \times 1 \times 1 \mathrm{~mm}$. As for Section IV of [21], a Gaussian pulse is employed and an observation point at a distance of 40 cells from the source is selected.

Fig. 4 illustrates the time and frequency domain waveforms of the normalized $E_{x}$ component recorded at the observation point as calculated by the trapezoidal model (dotted lines) and the Cartesian model of [21] (solid lines). We would expect some variation in the magnitudes of these recorded waveforms of the Cartesian and trapezoidal models, since the trapezoidal model includes curvature. Note, however, from Fig. 4 that both models correctly produce the slow whistler mode below the electron cyclotron frequency, $1.06 \times 10^{10} \mathrm{rad} / \mathrm{s}[21]$, and a resonance at the cyclotron frequency. Further, both models clearly demonstrate the LH- and RH-circularly polarized modes in the frequency domain, and both time-domain waveforms demonstrate the slow whistler mode as a low frequency oscillation arriving at about 600 time steps. Thus, the 3-D trapezoidal FDTD plasma model produces numerical results that agree with plasma theory and correctly demonstrate the anisotropic features of magnetized plasma.

\section{Global Validation Description}

The 3-D Cartesian-coordinate FDTD plasma code of [21] has been previously validated for accurate calculation of EM wave propagation in the magnetized plasma. This includes validation tests of Faraday rotation and whistler-mode propagation. Here, in the interest of testing the plasma's accuracy on a global scale, the previous propagation attenuation study of [22] is repeated but with the magnetized ionospheric plasma algorithm. Note that this validation test permits comparison of the propagation attenuation as calculated from two vastly different formulations. It will compare the results of (1) a global FDTD model having the ionosphere represented by a simple exponential conductivity profile that is accounted for through the traditional updating coefficients of the FDTD grid electric fields; and the results of (2) a global FDTD model having the ionosphere represented by the magnetized cold plasma that is accounted for via coupling of

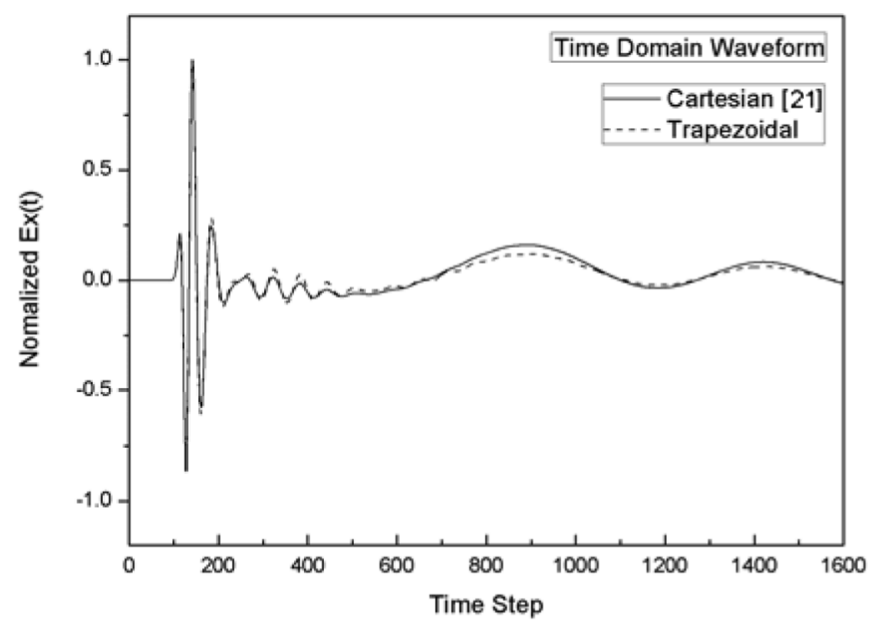

(a)

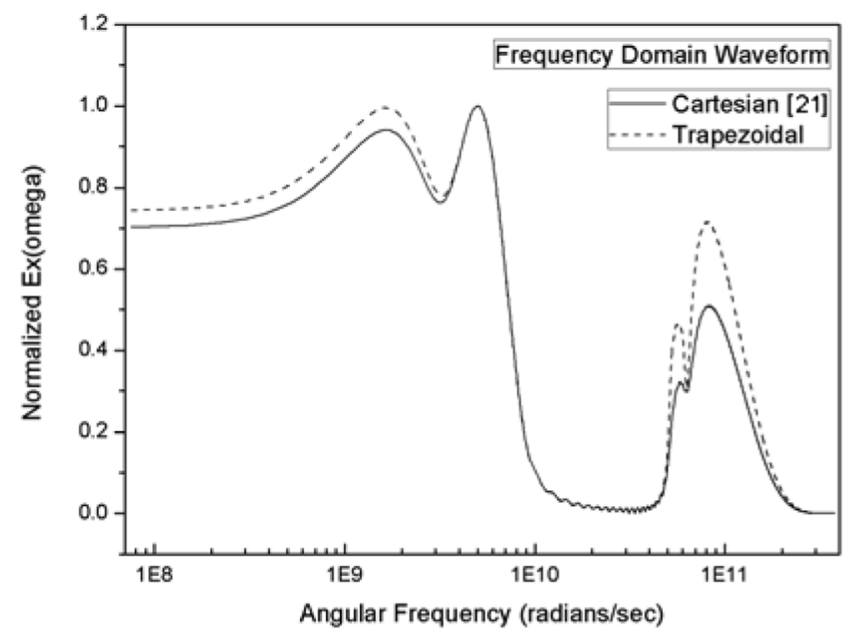

(b)

Fig. 4. Waveforms calculated by the trapezoidal model (dashed line) and the Cartesian model (solid line) for a Gaussian-pulsed plane wave propagation in the plasma with an applied magnetic field. (a) Time domain and (b) frequency domain.

the Maxwell's FDTD equations with current equations derived from the Lorentz equation of motion.

Following the general validation procedure of [22], global topographic and bathymetric data are taken from the NOAANGDC "Global Relief CD-ROM" [29] and incorporated into the FDTD Earth-magnetized ionosphere plasma model. These data are mapped onto the 3-D space lattice with an assumed resolution of $40 \times 40 \times 5 \mathrm{~km}$ at the equator. For the lithosphere, the same conductivity values of [22] are assigned depending upon whether the space lattice point is located directly below an ocean or within a continent. And finally, for the low-altitude atmosphere, the same exponential conductivity profile of [22] is also assumed.

For the magnetized ionospheric plasma assigned at high altitudes, the position-and time-dependent density profiles of electrons and ions can be obtained, for example, from the International Reference Ionosphere (IRI) [30]. However, for the general validation study in this paper, exponential profiles for the particle densities and the collision frequencies as proposed in [31] are utilized. Finally, the global geomagnetic field data is 


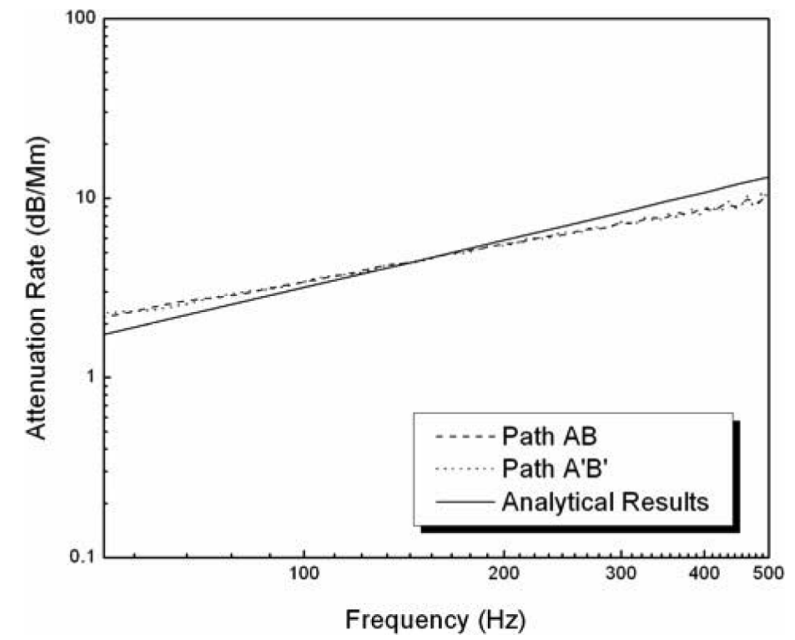

Fig. 5. Comparison between the FDTD-calculated ELF propagation attenuation versus frequency over paths $A B$ and $A^{\prime} B^{\prime}$ and the analytical results $\left(B_{0}=0\right)$.

obtained from the International Geomagnetic Reference Field (IGRF) model [32] and is mapped onto the FDTD mesh.

Continuing with the general procedure of [22], the new model is excited with a vertical, 5-km-long current pulse having a Gaussian time-waveform with a $1 / \mathrm{e}$ full-width of $480 \Delta t$, where $\Delta t=3.0 \mu \mathrm{s}$. To ensure a smooth onset of the excitation, the temporal center of this pulse is at $960 \Delta t$. This current pulse is located just above the Earth's surface on the equator at longitude $47^{\circ} \mathrm{W}$.

\section{Global Validation Results}

Following the same procedure as in [22], the surface radial $E$-field is recorded at observation points $A$ and $A^{\prime}$ on the Earth's surface at the equator directly East and West of the source at 1/4; of the distance to the antipode, and at points $B$ and $B^{\prime}$ at the equator directly East and West of the source at $1 / 2$; of the distance to the antipode. The propagation attenuation versus frequency for these FDTD time waveforms is then calculated by forming the ratio of the discrete Fourier transforms (DFTs) of the time-waveforms.

First, we consider the case wherein the background magnetic field $\left(B_{0}\right)$ is set to zero (resulting in an unmagnetized ionospheric plasma and, therefore, an isotropic ionosphere). Fig. 5 compares the FDTD-calculated ELF propagation attenuation versus frequency over paths $A B$ and $A^{\prime} B^{\prime}$ with the analytical results of [33]. Over the frequency range $50-500 \mathrm{~Hz}$, the FDTD-computed propagation attenuation values show close agreement with the analytical results, and the East and West FDTD-calculated attenuation rate are nearly identical to each other. However, it can be seen from Fig. 5 that the slope of the FDTD-calculated curve is slightly different from that of the analytical results. A complete agreement is not necessarily unexpected because the FDTD model is much more comprehensive than the analytical calculations by including the physics of the magnetized ionospheric plasma in addition to the Earth's topography, etc. Another possibility for the slope difference is that the ionospheric electron density and collision frequency

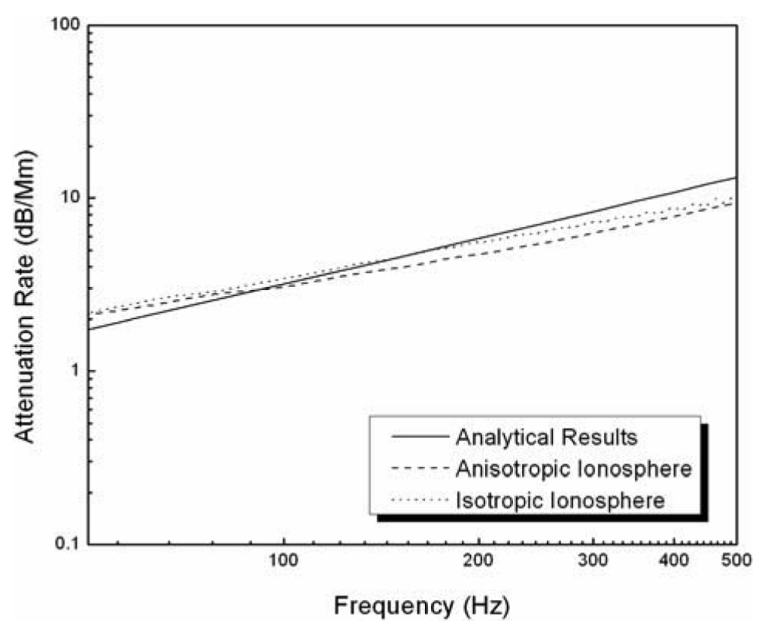

Fig. 6. Comparison between the FDTD-calculated ELF propagation attenuation versus frequency over path $A B$ for both the isotropic and anisotropic ionosphere cases and the analytical results.

profiles employed in the FDTD mesh are not sufficient or general enough to agree with the analytical attenuation rate.

Next, the background magnetic field is set to the geomagnetic field (resulting in a magnetized ionospheric plasma and, therefore, an anisotropic ionosphere). Initially, this global magnetized ionospheric plasma algorithm is employed at altitudes above $60 \mathrm{~km}$. However, the code is found to be unstable after about 7,000 time steps, despite utilizing double precision. This instability is believed to be caused by the accumulation of errors resulting from $\nu \cdot \Delta t$ being significantly greater than unity [21] at low altitudes, where $\nu$ is the particle collision frequency. One option to remedy this problem is to lower the time step $\Delta t$ so that $\nu \cdot \Delta t$ is always less than unity. However, this would require a time step at nearly 100 times smaller than that permitted by the Courant limit, resulting in a tediously long simulation run-time even with today's advanced super-computing capabilities. Instead, by raising the interface between the isotropic ionosphere and magnetized ionospheric plasma from $60 \mathrm{~km}$ to $80 \mathrm{~km}$, the code is found to be stable because at and above $80 \mathrm{~km}, \nu \cdot \Delta t$ is always less than unity.

Fig. 6 illustrates comparison between the FDTD-calculated ELF propagation attenuation versus frequency over path $A B$ as calculated from the global Earth-magnetized ionosphere model. Also shown in Fig. 6 are the path AB isotropic ionosphere results of Fig. 5 and the analytical results. In Fig. 6, there is very close agreement between all three curves, and the slopes of the path $\mathrm{AB}$ isotropic and anisotropic ionosphere FDTD results are nearly identical.

Note that the advantage of the model developed herein is not that it can provide results on the fly, especially when run in the future at even higher resolutions and to higher altitudes. For example, at the present resolution, running this model on 64 processing cores for about $\sim 80000$ time steps requires about $48 \mathrm{~h}$.

Instead, the advantage of this model is that it provides a very comprehensive physics analysis for EM wave phenomena in the Earth-ionosphere system while accounting for the highly complex geometry and position-dependent properties of the Earth- 
ionosphere system. Indeed, at higher frequencies, this model could represent a paradigm shift from ray-tracing calculations that provide only an approximation of the dominant path of energy flow and its characteristics through the ionosphere, to full-vector Maxwell's equations solutions of EM propagation not only through the highly complex ionosphere, but also of the complex scattering and diffraction of those waves as they reach the Earth's surface and/or satellite antennas.

\section{CONCLUSION AND ONGOING WORK}

A new global FDTD model of the Earth-ionosphere system accounting for the magnetized ionospheric plasma has been presented. In addition to a local validation study of the latitude-longitude FDTD plasma model that demonstrates correct calculations of electromagnetic propagation in magnetized plasma, a global validation study has also been performed for calculating the propagation characteristics at ELF frequencies.

Naturally, by accounting for more physics, the computational demands of this model are larger that the global model having only an isotropic conductivity profile. New $J$ variables have been introduced, new coefficients for the $E$ and $J$ updates must be stored, and more spatial averaging and updating calculations must be performed at each time step. This may limit the feasibility of extending the newly created model described in this paper to significantly higher altitudes and higher frequencies unless a very massively parallel computing cluster is utilized. On the other hand, computational resources over the past several decades have improved dramatically, providing a means to progress from 2-D Earth-ionosphere models to fully 3-D global Earth-ionosphere models within a 5-year time span, and now after 6 years to 3-D global Earth-ionosphere models that accommodate the magnetized ionospheric plasma. As such, we expect that even global simulations of EM propagation at frequencies approaching those used for high-frequency communications, over-the-horizon radar, and possibly even satellite communications is likely to become routine using FDTD in the future. Also, this model even opens up the possibility of modeling both the high-power, high-frequency HAARP transmitter along with the induced low-frequency EM wave propagation within a single, self-consistent model.

The new global models described herein thus represent a paradigm shift from ray-tracing codes that have been favored for many decades but that provide only an approximation of the dominant path of energy through the ionosphere. FDTD can provide in a single, self-consistent model the full-vector Maxwell's equations solutions of EM propagation not only through the highly complex ionosphere, but also of the complex scattering and diffraction of those waves as they reach the Earth's surface and/or satellite antennas.

Further, accounting for the magnetized plasma paves the way for coupling the FDTD Maxwell's equations models to other geophysical models, such as Naval Research Lab's SAMI 3 [20], in order to create a multiphysics simulator. In fact, this work is ongoing in the research lab of the authors.

\section{REFERENCES}

[1] K. Yee, "A numerical solution of initial boundary value problems involving Maxwell's equations in isotropic media," IEEE Trans. Antennas Propagat., vol. AP-14, no. 4, pp. 302-307, May 1966.
[2] A. Taflove and S. C. Hagness, Computational Electromagnetics: Finite-Difference Time-Domain Method, 3rd ed. Norwood, MA: Artech House, 2005.

[3] J. J. Simpson and A. Taflove, "A review of progress in FDTD Maxwell's equations modeling of impulsive sub-ionospheric propagation below $300 \mathrm{kHz}$," IEEE Trans. Antennas Propagat., vol. 55, no. 6, pp. 1582-1590, Jun. 2007, Special Issue on Electromagnetic Wave Propagation in Complex Environments: A Tribute to Leopold Benno Felsen.

[4] J. J. Simpson, "Current and future applications of full-vector 3-D Maxwell's equations FDTD global earth-ionosphere waveguide models," Surv. Geophys., vol. 30, no. 2, pp. 105-130, 2009.

[5] M. Thèvenot, J. P. Bérenger, T. Monedière, and F. Jecko, "A FDTD scheme for the computation of VLF-LF propagation in the anisotropic earth-ionosphere waveguide," Ann. Telecommun., vol. 54, pp. 297-310, 1999.

[6] J. P. Berenger, "FDTD computation of VLF-LF propagation in the Earth-ionosphere waveguide," Ann. Telecommun., vol. 57, no. 11-12, pp. 1059-1090, 2002.

[7] S. A. Cummer, "Modeling electromagnetic propagation in the Earthionosphere waveguide," IEEE Trans. Antennas Propagat., vol. 48, no. 9, pp. $1420-1429$, Sep. 2000.

[8] Y. Todoroki, S. Maekawa, T. Yamauchi, T. Horie, and M. Hayakawa, "Solar flare induced D region perturbation in the ionosphere, as revealed from a short-distance VLF propagation path," Geophys. Res. Lett., vol. 34, no. L03103, 2007.

[9] Y. T. Tanaka, T. Terasawa, M. Yoshida, T. Horie, and M. Hayakawa, "Ionospheric disturbances caused by SGR $1900+14$ giant gamma ray flare in 1998: Constraints on the energy spectrum of the flare," $J$. Geophys. Res., vol. 113, no. A07307, 2008.

[10] M. Hayakawa and T. Otsuyama, "FDTD analysis of ELF wave propagation in in homogencous sub ionospheric waveguide models," Appl. Computat. Electromagn. Soc. J., vol. 17, no. 3, pp. 239-244, 2002.

[11] J. J. Simpson and A. Taflove, "Three-dimensional FDTD modeling of impulsive ELF antipodal propagation and Schumann resonance of the Earth-sphere," IEEE Trans. Antennas Propagat., vol. 52, no. 2, pp. 443-451, Feb. 2004.

[12] H. Yang and V. P. Pasko, "Three-dimensional finite-difference timedomain modeling of the Earth-ionosphere cavity resonances," Geophys. Res. Lett., vol. 32, no. L03114, 2005.

[13] J. J. Simpson and A. Taflove, "A novel ELF radar for major oil deposits," IEEE Geosci. Remote Sens. Lett., vol. 3, no. 1, pp. 36-39, Jan. 2006 .

[14] J. J. Simpson and A. Taflove, "ELF radar system proposed for localized D-region ionospheric anomalies," IEEE Geosci. Remote Sens. Lett., vol. 3, no. 4, pp. 500-503, Oct. 2006.

[15] J. J. Simpson and A. Taflove, "Electrokinetic effect of the Loma Prieta earthquake calculated by an entire-Earth FDTD solution of Maxwell's equations," Geophys. Res. Lett., vol. 32, no. L09302, 2005.

[16] J. J. Simpson, "On the possibility of high-level transient coronal mass ejection-induced ionospheric current coupling to electric power grids," J. Geophys. Res.-Space Phys., vol. 116, no. A11308, 2011.

[17] M. L. Stone, L. E. Bird, and M. Balser, "A Faraday rotation measurement on the ionospheric perturbation produced by a burning rocket," J. Geophys. Res., vol. 69, no. 5, pp. 971-978, 1964.

[18] V. S. Sonwalkar, X. Chen, J. Harikumar, D. L. Carpenter, and T. F. Bell, "Whistler-mode wave-injection experiments in the plasma sphere with a radio sounder," J. Atmos. Solar-Terrestrial Phys., vol. 63, pp. 1199-1216, 2001.

[19] U. S. Inan, D. Piddyachiy, W. B. Peter, J. A. Sauvaud, and M. Parrot, "DEMETER satellite observations of lightning-induced electron precipitation," Geophys. Res. Lett., vol. 34, no. L07103, 2007.

[20] J. D. Huba, G. Joyce, J. Krall, C. L. Siefring, and P. A. Bernhard, "Self-consistent modeling of equatorial dawn density depletions with SAMI3," Geophys. Res. Lett., vol. 37, no. L03104, 2010.

[21] Y. Yu and J. J. Simpson, "An E-J collocated 3-D FDTD model of electromagnetic wave propagation in magnetized cold plasma," IEEE Trans. Antennas Propagat., vol. 58, no. 2, pp. 469-478, Feb. 2010.

[22] J. J. Simpson and A. Taflove, "Three-Dimensional FDTD modeling of impulsive ELF propagation about the Earth-sphere," IEEE Trans. Antennas Propagat., vol. 52, no. 2, pp. 443-451, Feb. 2004.

[23] J. J. Simpson, R. P. Heikes, and A. Taflove, "FDTD modeling of a novel ELF radar for major oil deposits using a three-dimensional geodesic grid of the Earth-ionosphere waveguide," IEEE Trans. Antennas Propagat., vol. 54, no. 6, pp. 1734-1741, Jun. 2006. 
[24] W. Hu and S. A. Cummer, "An FDTD model for low and high altitude lightning-generated EM fields," IEEE Trans. Antennas Propagat., vol. 54, no. 5, pp. 1513-1522, May 2006.

[25] Y. Yu and J. J. Simpson, "A magnetic field-independent absorbing boundary condition for magnetized cold plasma," IEEE Antennas Wireless Propagat. Lett., vol. 10, pp. 294-297, 2011.

[26] J. H. Lee and D. K. Kalluri, "Three dimensional FDTD simulation of electromagnetic wave transformation in a dynamic inhomogeneous magnetized plasma," IEEE Trans. Antennas Propagat., vol. 47, no. 7, pp. 1148-1151, Jul. 1999.

[27] J. L. Young, A. Kittichartphayak, Y. M. Kwok, and D. Sullivan, "On the dispersion errors related to (FD) ${ }^{2}$ TD type schemes," IEEE Trans. Microw. Theory Tech., vol. 43, pp. 1902-1909, 1995.

[28] S. Liu and S. Liu, "Runge-Kutta exponential time differencing FDTD method for anisotropic magnetized plasma," IEEE Antennas Wireless Propagat. Lett., vol. 7, pp. 306-309, 2008.

[29] Global Relief CD-ROM, NOAA-NGDC.

[30] [Online]. Available: http://ccmc.gsfc.nasa.gov/models/modelinfo.php?model=IGRF

[31] J. R. Wait and K. P. Spies, Characteristics of the Earth-Ionosphere Waveguide for VLF Radio Waves. Boulder, CO: National Bureau of Standards, 1964.

[32] [Online]. Available: http://ccmc.gsfc.nasa.gov/modelweb/models/ iri_vitmo.php

[33] P. Bannister, "ELF propagation update," IEEE J. Ocean. Eng., vol. 0E-9, no. 3, pp. 179-188, Apr. 1984.

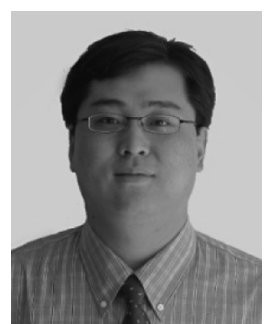

Yaxin Yu (M'09) received the B.S. degree in physics from Northwest University, Xi'an, China, in 2000; the M.S. degree in optics from Nankai University, Tianjin, China, in 2003; and the M.S. and Ph.D. degrees in electrical engineering from the University of New Mexico, Albuquerque, in 2006 and 2011, respectively.

Upon completion of his Ph.D. studies, he joined the Institute of High Performance Computing (IHPC), Agency for Science, Technology and Research (A*STAR), Singapore, where he is currently a Research Scientist in the Electronics and Photonics Department. His research interests include modeling of electromagnetic scattering and propagation both in local anisotropic media and in the global Earth-ionosphere system, modeling and simulation of photonic and plasmonic devices and circuits, electromagnetic compatibility, and high-performance computing.

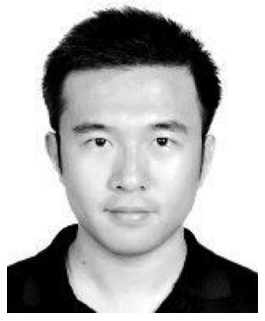

Jiajun Niu (S'10) received the B.S. and M.S. degrees from Shanghai Jiaotong University, Shanghai, China, in 2005 and 2008, respectively. He is currently pursuing the Ph.D. degree in the Electrical and Computer Engineering Department, University of New Mexico, Albuquerque.

His research interests include finite-difference time-domain (FDTD) solution of Maxwell's equations and its applications in simulating controlled source electromagnetic detection of sub-seafloor hydrocarbon reservoir, multiphysics modeling for electromagnetic wave propagation in the Earth-ionosphere system, as well as parallel programming on computer clusters.

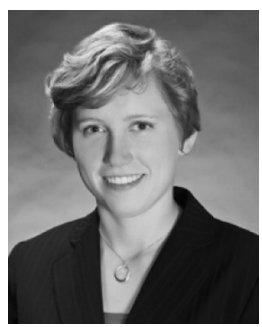

Jamesina J. Simpson (S'01-M'07) received the B.S. and Ph.D. degrees in electrical engineering from Northwestern University, Evanston, IL, in 2003 and 2007, respectively.

In 2007, she joined the Electrical and Computer Engineering Department, University of New Mexico, Albuquerque, as a tenure-track Assistant Professor. As of July 2012, she is an Associate Professor in the Electrical and Computer Engineering Department, University of Utah, Salt Lake City. Her research lab encompasses the application of the full-Maxwell's equations finite-difference time-domain (FDTD) method to model a wide variety of scientific and engineering applications.

Dr. Simpson is a recipient of the National Science Foundation Graduate Research Fellowship and the IEEE AP-S and MTT-S Graduate Research Awards. In 2010, she received an NSF CAREER award and in 2012 she received the Donald G. Dudley, Jr. Undergraduate Teaching Award of the IEEE AP-S. 